\title{
Regulation of Synaptic Plasticity and Synaptic Vesicle Dynamics by the PDZ Protein Scribble
}

\author{
John P. Roche, ${ }^{*}$ Mary C. Packard, ${ }^{*}$ Stephanie Moeckel-Cole, and Vivian Budnik \\ Department of Biology, Molecular and Cellular Biology Program, University of Massachusetts, Amherst, Massachusetts \\ 01003
}

The Drosophila tumor suppressor Scribble (Scrib) is a PDZcontaining protein required for maintaining epithelial cell polarity. At the larval neuromuscular junction, Scrib colocalizes and indirectly interacts with another tumor suppressor and PDZ protein, Discs-Large (Dlg). Previous studies demonstrate that Dig is critical for development of normal synapse structure and function, as well as for normal synaptic Scrib localization. Here we show that Scrib is also an important regulator of synaptic architecture and physiology. The most notable ultrastructural defect in scrib mutants is an increase in the number of synaptic vesicles in an area of the synaptic bouton thought to contain the reserve vesicle pool. Additionally, the number of active zones is reduced in scrib mutants. Functionally, the scrib syn- apse behaves relatively normally at low-frequency stimulation. However, several forms of plasticity at this synapse are drastically altered in the mutants. Specifically, scrib mutants exhibit loss of facilitation and post-tetanic potentiation, and faster synaptic depression. In addition, FM1-43 imaging of recycling synaptic vesicles shows that vesicle dynamics are impaired in scrib mutants. These results identify Scrib as an essential regulator of short-term synaptic plasticity. Taken together, our results are consistent with a model in which Scrib is required to sustain synaptic vesicle concentrations at their sites of release.

Key words: Drosophila; synapse; facilitation; PTP; vesicle recycling; scribble; PDZ; Dlg; FM1-43; calcium; Guk-holder
Scaffolding proteins are crucial for the correct localization of synaptic proteins (Koh et al., 2000; Sheng, 2001; Sheng and Sala, 2001). They are typically composed of multiple protein-binding domains and hence are important in forming a linked network of synaptic constituents and the underlying cytoskeleton. This linking of proteins is thought to be important for the spatial convergence of synaptic signaling mechanisms, for increased speed and accuracy of synaptic transmission, and for regulatory mechanisms required in synapse plasticity. Indeed, colocalization of transmembrane receptors with cytoplasmic signaling components via their interaction with scaffolding proteins has been documented in numerous studies. A prominent example is the mammalian scaffolding protein PSD-95, which is believed to link NMDA receptors to a Ras signaling pathway by simultaneously binding the $\mathrm{C}$ terminus of NMDA receptor subunits and a synaptic Ras-GTPase-activating protein SynGAP (Chen et al., 1998; Kim et al., 1998).

Studies in both mammalian and insect preparations have identified members of the PSD-95 family, including the Drosophila protein Discs-Large (Dlg), as central elements in the maturation and plasticity of glutamatergic synapses (Lahey et al., 1994; Kornau et al., 1995; Kim and Sheng, 1996). At these synapses, PSD-95 and Dlg bind and localize a number of synaptic constit-

Received March 1, 2002; revised May 1, 2002; accepted May 13, 2002.

This work was supported by National Institutes of Health Grants RO1 NS 37061 and R01 NS42629 to V.B. We thank Drs. Michael Gorczyca, Catalina Ruiz-Canada, and Dennis Mathew for careful reading of this manuscript. We also thank the University of Massachusetts Imaging Facility for their support in electron microscopical and confocal studies, and in particular Lucy Yin for her help in the sectioning of EM preparations.

*J.P.R. and M.C.P. contributed equally to this work.

Correspondence should be addressed to Vivian Budnik, Biology Department and Molecular and Cellular Biology Program, 221 Morrill Science Center, University of Massachusetts at Amherst, Amherst, MA 01003. E-mail: vbudnik@bio.umass.edu. Copyright @ 12002 Society for Neuroscience 0270-6474/02/226471-09\$15.00/0 uents critical for initiation and maintenance of synaptic plasticity, including NMDA and kainate receptor subunits and the cell adhesion molecule Fasciclin II (Kornau et al., 1995; Niethammer et al., 1996; Thomas et al., 1997; Garcia et al., 1998). We have recently demonstrated that at synapses Dlg also interacts with a novel PDZ scaffolding protein, Scribble (Scrib), through simultaneous binding of both the guanylate kinase domain of Dlg and the PDZ2 domain of Scrib to the synaptic protein GUK-holder (Gukh) (Mathew et al., 2002).

Scrib is a 1731 amino acid cytoplasmic protein first characterized in epithelial cells where it plays a central role in maintaining apical polarity and in tumor suppression (Bilder and Perrimon, 2000; Bilder et al., 2000). Scrib contains several consensus binding motifs including a set of 16 leucine-rich repeats and 4 PDZ domains. In wild-type synapses, Scrib is tightly colocalized with Dlg, a protein associated with both presynaptic and postsynaptic membranes. However, loss-of-function mutations in $\mathrm{dlg}$ and $g u k h$ lead to a striking mislocalization of Scrib at the neuromuscular junction (NMJ) (Mathew et al., 2002).

Here we examined both the structure and physiology of scrib mutant synapses to understand the synaptic function of Scrib and to determine whether the defects in structural plasticity in $d l g$ mutants might result from the loss of synaptic Scrib function. Our studies show that in scrib mutants short-term synaptic plasticity is severely impaired. Ultrastructural analysis of scrib synapses, in vivo labeling of activity-dependent vesicle recycling, and functional studies using high-frequency stimulation are all consistent with a model by which vesicle dynamics are disrupted in scrib mutants. Our results point to a novel role for scaffolding proteins in sustaining synaptic vesicle concentrations at their sites of release during high-frequency stimulation, an important process involved in many forms of synaptic plasticity. 


\section{MATERIALS AND METHODS}

Flies. The following fly strains were used in these studies: (1) mutations in $s c r i b\left[s c r i b^{1}, s c r i b^{2}\right.$, and Df(3R)Tl-x, a deficiency of the scrib region (Bilder and Perrimon, 2000; Bilder et al., 2000)], (2) Gal4 driver strains BG487 and C380 (Koh et al., 1999), (3) UAS-Scrib (Bilder and Perrimon, 2000), and (4) the wild-type strain Canton S (CS).

Electron microscopy. For transmission electron microscopy and morphometric analysis of synapses, body wall muscles from different genotypes were processed as described previously (Jia et al., 1993; Torroja et al., 1999). Comparisons between genotypes were performed in animals processed for EM simultaneously, and in at least two separate experiments. Synaptic boutons from muscles 6 and 7 (segments A2 and A3) in early to mid third instars were serially sectioned, and the section of largest area (defined as the bouton midline) was used for quantitative analysis (Thomas et al., 1997; Koh et al., 1999). To determine vesicle density, vesicles at the bouton midline cross section were counted, and the number of vesicles was divided by the area of the cross section. To determine the number of vesicles in the readily releasable vesicle pool (RRP), semi-circumferences with radii at 100, 150, and $200 \mathrm{~nm}$ from the intersection of the $\mathrm{T}$-shaped active zone and the presynaptic membrane were traced (see Fig. $4 A$ ), and the number of vesicles within or intersected by each semi-circumference was counted. To determine subsynaptic reticulum (SSR) length, electron micrographs were printed at $30,000-60,000 \times$ the SSR traced, scanned, and analyzed using $\mathrm{NIH}$ Image as described previously (Budnik et al., 1996; Thomas et al., 1997). The number of preparations $(N)$ and number of boutons serially sectioned $(n)$ and analyzed for each genotype are in the legend to Figure 2.

Electrophysiology. All recordings were done in muscle 6, abdominal segment 2 or 3 , of wandering third instar larvae. Dissections were done in ice-cold HL-3 (Stewart et al., 1994) solution containing $300 \mu \mathrm{M} \mathrm{Ca}^{2+}$. HL-3 solution composition was as follows (in $\mathrm{mM}$ ): $\mathrm{NaCl} 70, \mathrm{KCl} 5$, $\mathrm{MgCl}_{2} 20, \mathrm{NaHCO}_{3} 10$, trehalose 5, HEPES 5, sucrose $115 . \mathrm{Ca}^{2+}$ was added at various concentrations as indicated in Results. HL-3 solution was superfused continuously through the recording chamber throughout the duration of the experiment. Recordings were done in voltage-clamp mode using an Axoclamp 2A amplifier (Axon Instruments), filtered at 1 $\mathrm{kHz}$, and digitized with an Instrutech ITC-16 computer interface using Pulse software (HEKA electronics). Both evoked and spontaneous events were analyzed with Mini Analysis software (Synaptosoft Inc.). Stimulation was performed with a Grass $\mathrm{S} 88$ stimulator via a $\mathrm{AgCl}_{2}$ wire in a glass suction electrode. Nerves were stimulated for $0.3 \mathrm{msec}$ at $1.5 \times$ a voltage sufficient for maximal synaptic current. Muscles were held at $-70 \mathrm{mV}$.

FM1-43 labeling. Larvae were dissected in HL-3 containing $0.1 \mathrm{~mm}$ $\mathrm{Ca}^{2+}$ (low- $\mathrm{Ca}^{2+}$ saline) and incubated in high $\mathrm{K}^{+} \mathrm{HL}-3$ saline, adjusted to maintain osmolarity (in $\mathrm{mM}$ ): $\mathrm{NaCl} 40, \mathrm{KCl} 90, \mathrm{CaCl}_{2} 1.5, \mathrm{MgCl}_{2} 20$, $\mathrm{NaHCO}_{3} 10$, trehalose 5, HEPES 5, sucrose 5, and containing $5 \mu \mathrm{M}$ FM1-43 for 2 min at room temperature. Samples were then washed three times with low $\mathrm{Ca}^{2+}$ saline for $5 \mathrm{~min}$ each and fixed for $10 \mathrm{~min}$ in $4 \%$ paraformaldehyde fixative. After washing with PBS, samples were mounted in Vectashield mounting medium and imaged using a Bio-Rad MRC 600 confocal. Samples were subsequently demounted and processed for anti-HRP immunocytochemistry to visualize the presynaptic arbor as in Thomas et al. (2000). For quantification of FM1-43 staining intensities, samples were processed simultaneously and imaged using the confocal microscope using identical acquisition parameters. Background intensity (noise) was measured in NIH Image by tracing a line of $100 \mu \mathrm{m}$ across the muscle in a region devoid of boutons and measuring mean intensity (in 0-256 gray scale). Signal intensity at boutons was determined by tracing four lines at $90^{\circ}$ to each other from the center of the bouton to the end of the bouton rim and measuring the maximum signal intensity (in a $0-256$ gray scale).

\section{RESULTS}

\section{Scrib mutants have altered synaptic structure, including abnormal vesicle distribution}

Previous studies have shown that mutations in $d l g$ lead to prominent defects in both synapse structure and function. At the ultrastructural level these defects include an increase in bouton size and number of active zones, as well as a poorly developed SSR, an elaborate folding of the postsynaptic membrane at the NMJ (Budnik, 1996; Thomas et al., 1997). Dlg is colocalized with the PDZ-protein Scrib (Fig. $1 A-C$ ), and mutations in $d l g$ also
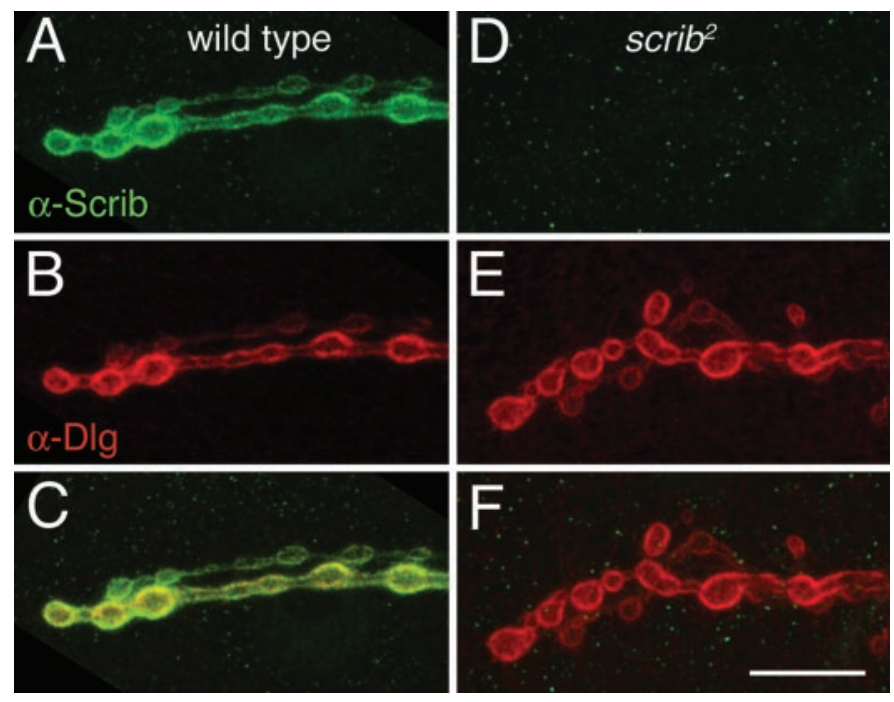

Figure 1. Scrib colocalizes with Dlg. $A-C$, Confocal micrographs of wild-type third instar larval NMJ at muscles 6 and 7 double labeled with anti-Scrib $(A)$ and anti-Dlg $(B)$. In $C$, both panels have been merged. $D-F$, NMJ in $s c r i b^{2}$ mutant showing absence of Scrib immunoreactivity $(D)$ and normal Dlg immunoreactivity $(E)$ in this mutant. In $F$, both $D$ and $E$ have been merged. Scale bar, $10 \mu \mathrm{m}$.

result in severe mislocalization of synaptic Scrib (Mathew et al., 2002). In contrast, although localization of Scrib to the NMJ is completely disrupted in scrib mutants, the localization of Dlg is not affected (Fig. $1 D-F$ ). Analysis of the NMJs in scrib mutants has shown that the general morphology is not affected (Mathew et al., 2002). We hypothesized that some of the defects in $d l g$ mutants might be the consequence of Scrib mislocalization. This hypothesis was tested by serially sectioning type I synaptic boutons in several scrib mutant allelic combinations and examining their ultrastructure using electron microscopy. We found that the synaptic structure in these mutants was drastically altered; however, these defects were quite distinct from those in $d l g$ mutants. One of the most prominent defects was an abnormally high density of synaptic vesicles (Fig. $2 A, B$ ). In wild type, synaptic vesicles are organized into at least two pools: a pool in direct proximity to the T-shaped active zones [thought to represent the readily releasable pool (RRP)], and a pool localized in a broad area at the periphery of the entire synaptic bouton [representing the reserve vesicle pool (RP)] (Kuromi and Kidokoro, 1998). Typically, the central region of the bouton is devoid of synaptic vesicles and contains endosomes and mitochondria, as well as other nonvesicular material (Fig. $2 A$ ). In contrast, boutons in the null allele $s c r i b^{2}$ and $s c r i b^{2} / D f$, but not those from the less severe allele $s c r i b^{1}$, were filled with synaptic vesicles and lacked an empty core (Fig. 2B). The number and area of mitochrondrial profiles, however, was unchanged in scrib mutant boutons (data not shown). Overall, in these mutants there was a significant increase in synaptic vesicle density, as measured by determining the total number of vesicles at the central cross section of the boutons divided by the area of this cross section (Fig. 3). In addition to this striking defect in vesicle distribution and density, many boutons contained morphologically abnormal vesicular material at the core (Fig. 2D, asterisk and inset).

To determine whether both the RRP and the RP were affected in scrib mutants, we counted the number of vesicles in an area 100, 150 , and $200 \mathrm{~nm}$ around the active zone, which likely encompasses the RRP (Fig. 4A). We found that the number of vesicles in these 

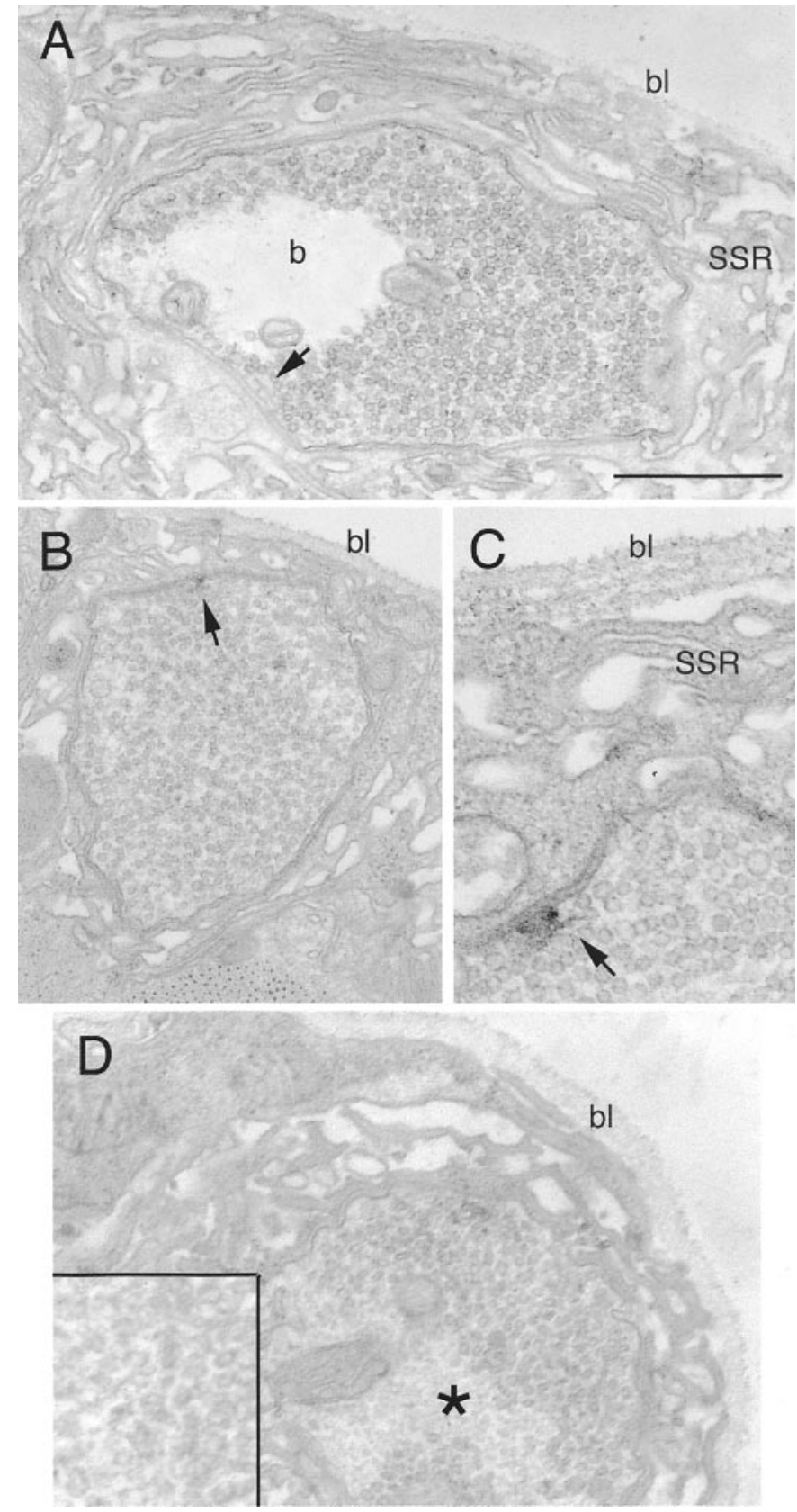

Figure 2. Synaptic ultrastructure is altered in scrib mutants. Electron micrographs of type I synaptic boutons in wild-type $(A)$ and $s c r i b^{2} / D f$ third instar $(B)$ larvae, showing the decrease in bouton size and increase in both vesicle density and basal lamina thickness in scrib mutants. $C$, High-magnification view of the synaptic basal lamina in a $s c r i b^{2}$ mutant showing several layers of basal lamina. $D$, Abnormal vesicle-like material at the core of $s c r i b^{2}$ mutant boutons is indicated with an asterisk and shown at higher magnification in inset. $b$, Bouton; $b l$, basal lamina; $S S R$, subsynaptic reticulum; arrow, active zones. Scale bar (shown in $A$ ): $A, B$, $D, 1 \mu \mathrm{m} ; C$, inset, $0.6 \mu \mathrm{m}$.

areas of $s c r i b^{2} / D f$ mutant boutons was not significantly different from wild type (Fig. $4 B-F$ ). Thus, it is the distribution and density of the RP that appear to be specifically affected in scrib mutants.

In addition to the defect in the RP, the average number of active zones in both $s c r i b^{2}$ and $s c r i b^{2} / D f$ was slightly lower than wild type, although this difference was statistically different only at $s c r i b^{2}$ homozygous boutons (Fig. 3). Unlike dlg mutants, the

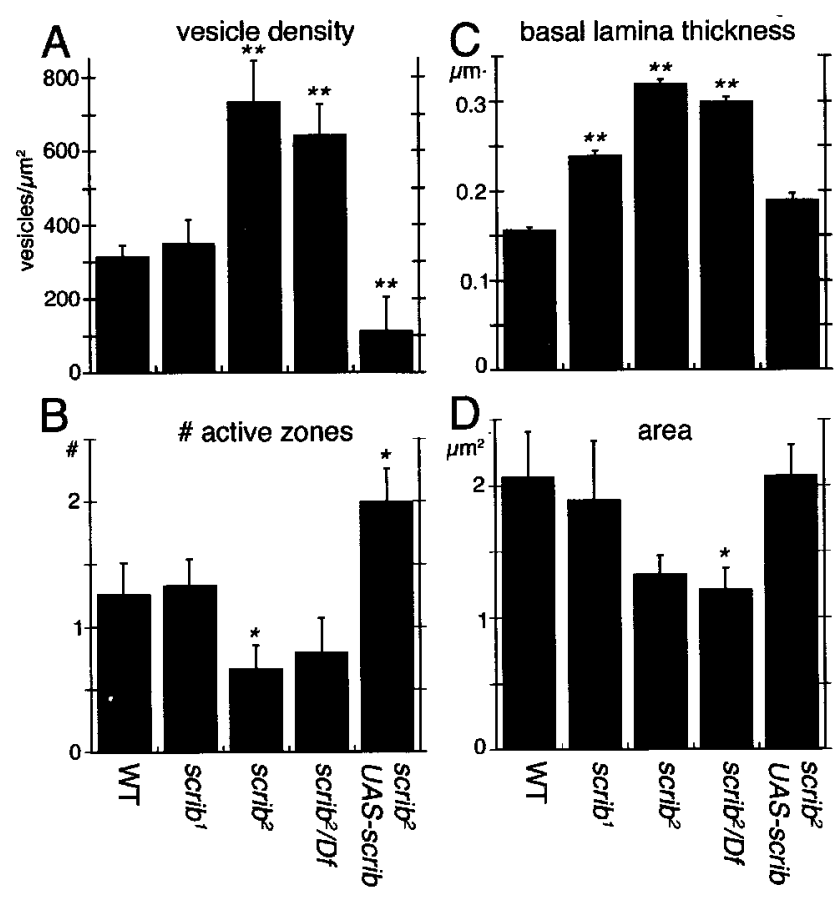

Figure 3. Morphometric analysis of synaptic boutons in wild-type and scrib mutants. $A$, Vesicle density (number of vesicles/cross-sectional area). $B$, Number of active zones per cross-sectional area. $C$, Basal lamina thickness. $D$, Cross-sectional area. Asterisk represents significant difference; Student's $t$ test; $p \leq 0.01$. All measurements were performed at the cross section of the bouton midline. The numbers of boutons quantified are 17 boutons in two wild-type preparations and 16 boutons in four $s c r i b^{2}$ preparations.

SSR appeared normal, and neither the number of SSR layers nor the SSR density was significantly different from wild-type controls (data not shown). This is in contrast to the observations in severe $d l g$ mutants, in which the number of active zones is increased several fold and the SSR length is reduced (Budnik et al., 1996; Thomas et al., 1997).

Another phenotype in scrib mutants was the presence of an abnormally thick basal lamina (Fig. $2 B-D$ ). In $\sim 30 \%$ of boutons examined, the muscle basal lamina appeared to be composed of several layers of normally sized basal lamina (Fig. 2C). However, at the majority of scrib mutant boutons, the basal lamina had a uniform electron density (Fig. 4B,D). These data suggest that $s c r i b$ is involved in the normal development of several aspects of synapse structure.

We next attempted to rescue the above mutant phenotypes by expressing transgenic Scrib in motor neurons and muscles of $s c r i b^{2}$ mutants, using the Gal4 drivers C380 and BG487 (Koh et al., 1999). We found that in these transgenic animals the density of synaptic vesicles was significantly decreased from the levels found in scrib mutants. In fact, the density of synaptic vesicles was significantly lower even than the densities in wild-type boutons. A similar effect was seen when analyzing the number of active zones, which was significantly higher than not only scrib mutants but also wild-type boutons (Fig. 3). These data suggest that expression of Scrib in the mutant background not only rescues the scrib mutant defect, but causes a new phenotype in the opposing direction and likely results from an overexpression of Scrib compared with wild type. In contrast, the defect in the basal lamina was only partially rescued by transgenic Scrib expression. Thus, both the synaptic vesicle density and the number of active zones 

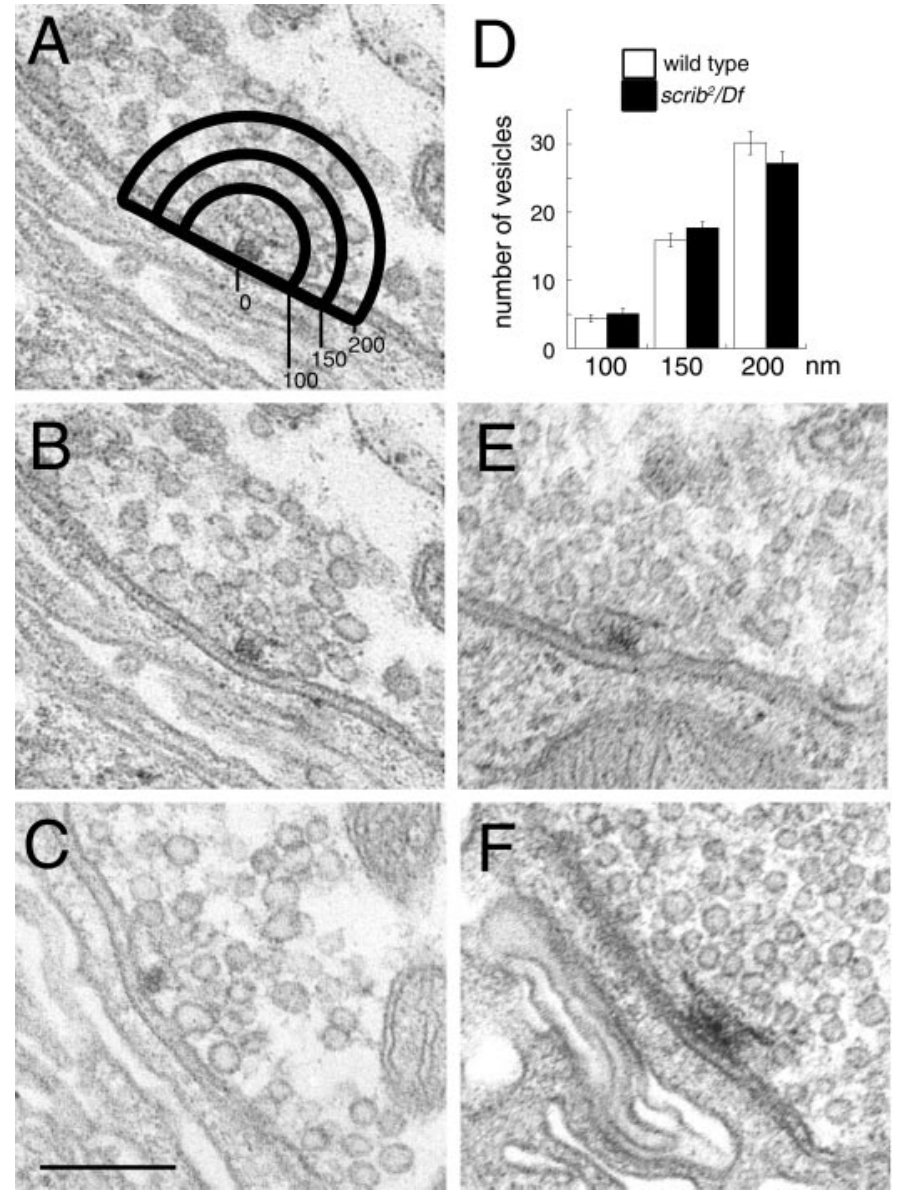

Figure 4. The RRP distribution is not affected by mutations in scrib. A, Active zone in a wild-type bouton indicating the areas around the active zone in which vesicles were counted. Numbers correspond to nanometers. $B, C, E, F$, Examples of active zones and associated vesicles in wild-type $(B, C)$ and $s c r i b^{2} / D f(E, F)$ boutons. $D$, Number of vesicles in the area described by a radius of 100,150 , and $200 \mathrm{~nm}$, and with a center in the middle of the active zone, in wild type (white) and scrib ${ }^{2} / D f$ (black). Numbers of active zones examined are 63 in $s c r i b^{2} / D f$ and 56 in wild-type boutons. Scale bar, $2 \mu \mathrm{m}$.

can be modified in either direction by manipulating the concentration of Scrib at the synapse.

\section{scrib mutants have abnormal spontaneous release but normal evoked release}

To understand the physiological significance of the structural defects observed in scrib mutants, we next examined synaptic function. We measured both evoked synaptic events [excitatory junctional currents (EJCs)] and spontaneous events [miniature EJCs (mEJCs)] by two-electrode voltage-clamp experiments in muscle 6 of third instar larvae. Changes in the frequency of miniature synaptic currents result from alterations in presynaptic function, caused by alterations either in the probability of release $\left(p_{\mathrm{R}}\right)$ or in the number of release sites. Conversely, changes in the amplitude of miniature events are generally considered to result from postsynaptic alterations, usually a change in receptor concentration or function. We found that in $s c r i b^{2} / D f$ larvae, there was a significant decrease in the frequency of miniature synaptic events (Fig. 5A,C). Additionally, there was a slight but significant decrease in the amplitude of miniature synaptic events (Fig. $5 A, B)$. Notably, presynaptic expression of transgenic Scrib using
A.
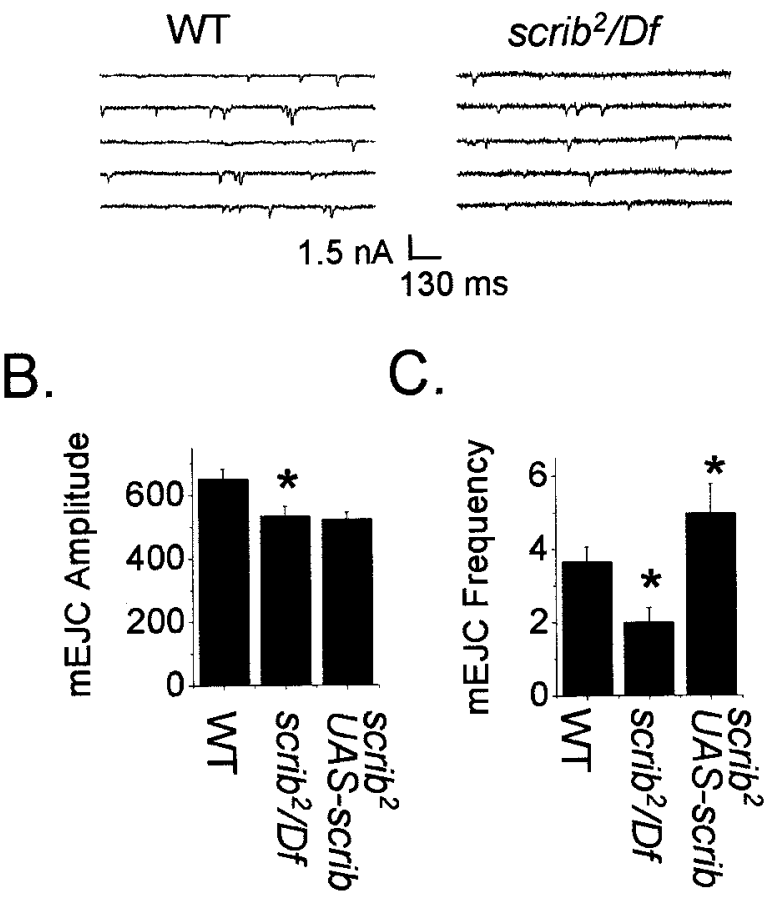

Figure 5. Mutation of scrib alters spontaneous synaptic currents. A, Representative spontaneous $\mathrm{mEJC}$ recordings from third instar larvae of wild type (left) or $s c r i b^{2 / D f}$ (right). Recordings were done in muscle 6, which was held at $-70 \mathrm{mV}$ in a $0.5 \mathrm{~mm} \mathrm{Ca}^{2+}$ saline solution. Spontaneous events were recorded for $1 \mathrm{~min}$ in $0.5 \mathrm{mM} \mathrm{Ca}{ }^{2+}$ saline solution, and the average amplitude $(B)$ and frequency $(C)$ of the spontaneous events were quantified. $n=17$ wild type, $8 \mathrm{scrib}^{2} / D f$, and $7 \mathrm{scrib}^{2}$ with presynaptic expression of transgenic Scrib (scrib ${ }^{2} U A S$-scrib) larvae. Asterisk represents significant difference; Student's $t$ test; $p \leq 0.05$.

the Gal4 driver C380 in the $s c r i b^{2}$ mutant background not only rescued the decrease in $\mathrm{mEJC}$ frequency but resulted in a significant increase in mEJC frequency compared with wild-type controls. This is in striking agreement with the ultrastructural studies, which show that expression of transgenic Scrib not only rescues the decrease in active zone number but results in a significant increase in the number of active zones compared with wild type. This result also is a strong indicator that, in this case, $\mathrm{mEJC}$ frequency is a reflection of the number of release sites.

Presynaptic expression of Scrib in the $s c r i b^{2} / D f$ background had no effect on mEJC amplitude (Fig. $5 B$ ), indicating that the reduced mEJC amplitude in $s c r i b^{2} / D f$ larvae arises from a postsynaptic mechanism.

We also measured evoked release by stimulating the segmental nerve innervating muscle 6 using a glass suction electrode. The nerve was stimulated with suprathreshold voltage at a frequency of $1 \mathrm{~Hz}$, and the resulting EJCs were recorded (Fig. 6A). Surprisingly, we found that there was no significant change in the amplitude of evoked EJCs when we compared $s c r i b^{2} / D f$ with wild type (Fig. $6 B$ ). There was a small but statistically insignificant increase in the quantal content of $s c r i b^{2} / D f$ larvae (Fig. $6 C$ ) resulting from the small decrease in $\mathrm{mEJC}$ size (Fig. $5 B$ ) and the equivalent EJC size (Fig. 6B). There was a slight divergence in wild-type and scrib mutant EJC amplitudes at low $\mathrm{Ca}^{2+}$ concentrations, with a significant difference only at $0.3 \mathrm{mM} \mathrm{Ca}^{2+}$ (Student's $t$ test; $p \leq 0.05$ ) (Fig. $6 D$ ). A double logarithmic plot of EJC 



Figure 6. Mutation of scrib has little effect on evoked synaptic currents. $A$, Representative recordings from third instar wild-type larvae (left) or $s c r i b^{2 / D f}$ (right). Recordings were done in muscle 6, which was held at $-70 \mathrm{mV}$ in $0.5 \mathrm{~mm}$ (top) or $1.0 \mathrm{~mm}$ (bottom) $\mathrm{Ca}^{2+}$ saline solution. The segmental nerve was stimulated with suprathreshold voltage at a frequency of $1 \mathrm{~Hz}$, and the resulting EJC was recorded. $B$, Quantification of the average EJC amplitude and quantal content $(C)$ at $0.5 \mathrm{~mm}$ $\mathrm{Ca}^{2+} . n=8$ wild type, $7 \mathrm{scrib}^{2} / \mathrm{Df}$, and $10 \mathrm{scrib}^{2}$, with presynaptic expression of transgenic Scrib (scrib ${ }^{2} U A S$-scrib) larvae. D, Quantification of the average EJC at various $\mathrm{Ca}^{2+}$ concentrations. Black filled squares represent the average EJC from wild-type larvae; open circles represent the average EJC from $s c r i b^{2} / D f$ larvae $(n=3-15)$. There was a significant difference only at one $\mathrm{Ca}^{2+}$ concentration tested, $0.3 \mathrm{~mm}$ (Student's $t$ test; $p \leq 0.05$ ). $E$, Fit to the linear portion of the $\mathrm{Ca}^{2+}$ concentration curve. Slopes obtained are $3.4 \pm 0.1$ for wild type and $2.8 \pm 0.4$ for $s c r i b^{2} / D f$.

amplitude in the linear portion of the $\mathrm{Ca}^{2+}$ concentration curve, an indicator of $\mathrm{Ca}^{2+}$ cooperativity (Dodge and Rahamimoff, 1967), reveals a slight change in the slope of the linear fit, from $3.4 \pm 0.1$ for wild type to $2.8 \pm 0.4$ for $s c r i b^{2} / D f$ (Fig. $6 E$ ). Thus, vesicle release in scrib2/Df is slightly more sensitive to external $\mathrm{Ca}^{2+}$ levels than wild-type larvae. However, this change in sensitivity is very small in comparison to other Drosophila mutations that alter the $\mathrm{Ca}^{2+}$ sensitivity of vesicle release (Zhong and $\mathrm{Wu}$,
1991; Rohrbough et al., 1999), perhaps a result of a mislocalization of release machinery with respect to cytoplasmic $\mathrm{Ca}^{2+}$ rather than a complete elimination of a critical component of the $\mathrm{Ca}^{2+}$ sensing process.

\section{scrib mutants have altered short-term synaptic plasticity}

The similarity of evoked responses was surprising in view of the drastic changes in the ultrastructure and the decrease in spontaneous release frequency at synapses of the scrib mutant larvae. We next tested the response to high-frequency stimulation to determine whether underlying defects may be uncovered during conditions in which the speed and accuracy of vesicle dynamics play a more crucial role. In wild type at $0.5 \mathrm{mM} \mathrm{Ca}^{2+}, 10 \mathrm{~Hz}$ stimulation results in short-term facilitation of the synaptic current (Fig. 7A). This is thought to be caused by increased vesicular release resulting from residual $\mathrm{Ca}^{2+}$ remaining in the neuronal cytoplasm from previous depolarizations, acting on unknown targets to increase vesicle release (Zhong and Wu, 1991; Zucker, 1999; Zucker and Regehr, 2002). In contrast to wild type, facilitation was severely reduced or absent in $s c r i b^{2} / D f$ mutants (Fig. 7 $A, B$ ), and unlike some other mutants that do not exhibit facilitation (Broadie et al., 1997; Rohrbough et al., 1999, 2000), the baseline transmission of $s c r i b^{2} / D f$ was equivalent to wild type at $0.5 \mathrm{~mm} \mathrm{Ca}^{2+}$. Presynaptic expression of transgenic Scrib restored the response of the synapse to high-frequency stimulation (Fig. 7 $A, B$ ).

To test whether the effect seen at $0.5 \mathrm{mM} \mathrm{Ca}^{2+}$ resulted from the specific inability to show facilitation or was a more global defect in synaptic vesicle replenishment at high stimulation frequencies, we tested the synaptic response at higher $\mathrm{Ca}^{2+}$ concentrations $(1.0 \mathrm{mM})$, where no facilitation is seen in wild-type larvae. At $1.0 \mathrm{~mm} \mathrm{Ca}{ }^{2+}$, little change in EJC amplitude is seen after switching to $10 \mathrm{~Hz}$ stimulation frequencies (Fig. 7C,D), presumably because of a balance between the number of vesicles released and the resupply of the RRP. In contrast to the maintained EJC amplitude seen in wild-type larvae at $10 \mathrm{~Hz}$, a significant depression is seen in $s c r i b^{2} / D f$ mutants, becoming apparent immediately after the first few stimuli (Fig. 7C,D). Thus, high-frequency stimulation causes $s c r i b^{2} / D f$ mutant synapses to exhibit both a lack of facilitation at low $\mathrm{Ca}^{2+}$ concentrations and faster synaptic depression at higher $\mathrm{Ca}^{2+}$ concentrations. These data suggest that $s c r i b^{2} / D f$ is defective not in the ability to exhibit facilitation but rather in the ability to resupply the RRP during high-frequency firing.

Another form of activity-dependent short-term plasticity exhibited at the Drosophila NMJ is post-tetanic potentiation (PTP). After a short train $(30 \mathrm{sec})$ of high-frequency $(10 \mathrm{~Hz})$ stimuli, the EJC is potentiated for a period of 3-4 min relative to EJCs before the tetanus. The amplitude of wild-type EJCs is potentiated more than twofold after the train of high-frequency stimulation (Fig. $8 A, B$ ). $s c r i b^{2} / D f$, in contrast, shows only a modest potentiation after the same stimulation protocol. Thus, multiple forms of activity-dependent plasticity are altered at the scrib synapse.

\section{scrib mutants have altered vesicle cycling}

The alteration in short-term plasticity observed in response to high-frequency stimulation in scrib mutants, combined with the ultrastructural observations showing a remarkable increase in vesicle density, suggest that vesicle dynamics might be affected in the mutants. For example, if the rapid recruitment of vesicles to 


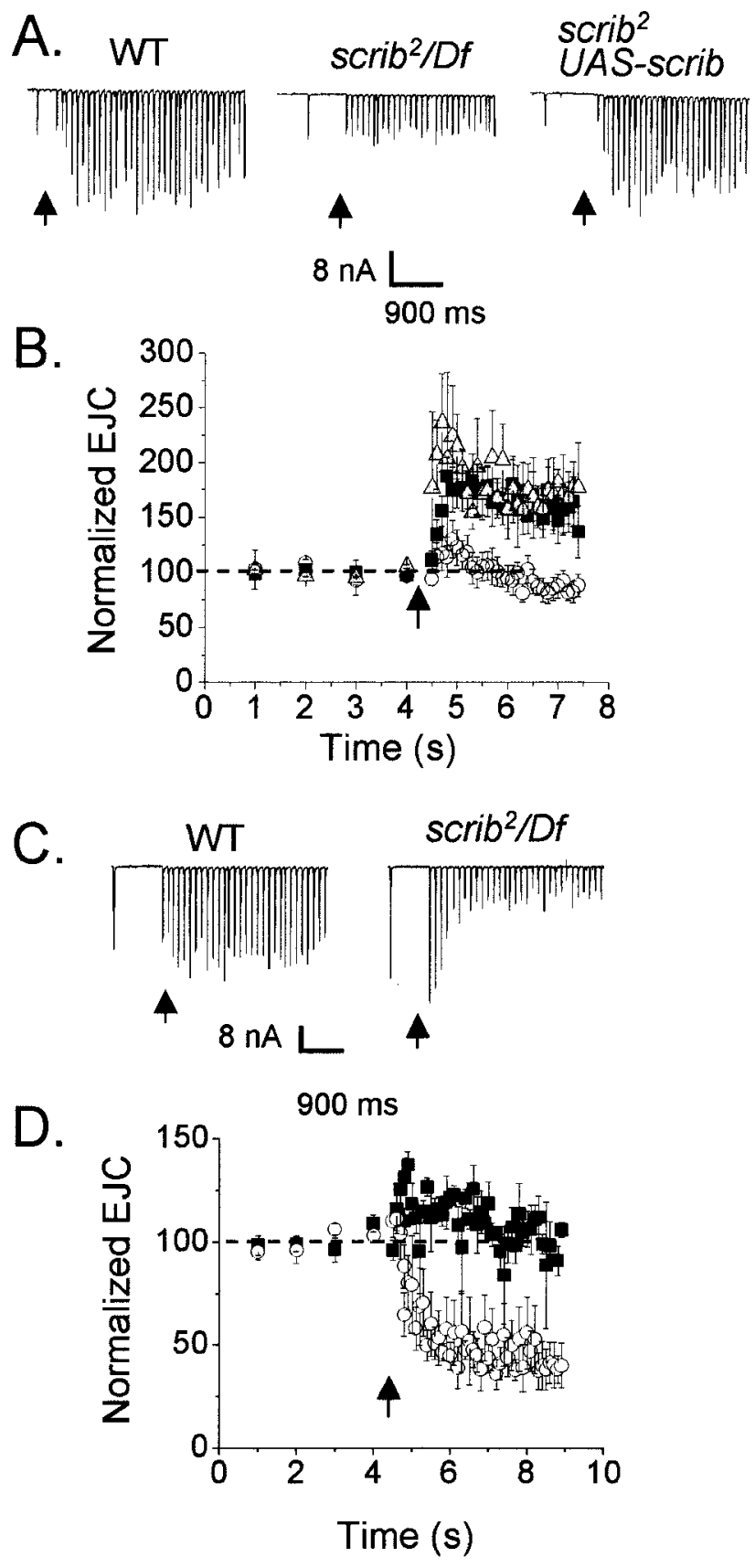

Figure 7. scrib mutants exhibit reduced synaptic current amplitude during high-frequency stimulation. $A$, Representative currents recorded from wild type (left), scrib ${ }^{2} / D f$ (middle), and $s c r i b^{2}$ with presynaptic expression of transgenic Scrib (scrib ${ }^{2} U A S$-scrib) larvae (right) at 1 and $10 \mathrm{~Hz}$ in 0.5 $\mathrm{mm} \mathrm{Ca}^{2+} . B$, Average response to high-frequency stimulation for wild type (black filled squares; $n=14)$, scrib $^{2} / D f$ (open circles; $\left.n=16\right)$, and scrib ${ }^{2} U A S$-scrib (gray-filled triangles; $n=4$ ) in $0.5 \mathrm{mM} \mathrm{Ca}^{2+}$. Dashed line represents normalized level of control $(100 \%)$. $C$, Representative currents recorded from wild-type (left) and $s c r i b^{2} / D f($ right $)$ larvae at 1 and $10 \mathrm{~Hz}$ in $1.0 \mathrm{~mm} \mathrm{Ca}^{2+} . D$, Average response to high-frequency stimulation for wild type (black filled squares; $n=4$ ) and scrib $/ D f$ (open circles; $n=4$ ) in $1.0 \mathrm{mM} \mathrm{Ca}^{2+}$. Dashed line represents normalized level of control (100\%). Arrow denotes switch from 1 to $10 \mathrm{~Hz}$ stimulation frequency.

the RRP is altered, then synaptic transmission is expected to be normal at low stimulation frequencies, because at these frequencies the RP does not contribute to vesicle release. However, at high frequencies the RRP size must be maintained by rapid
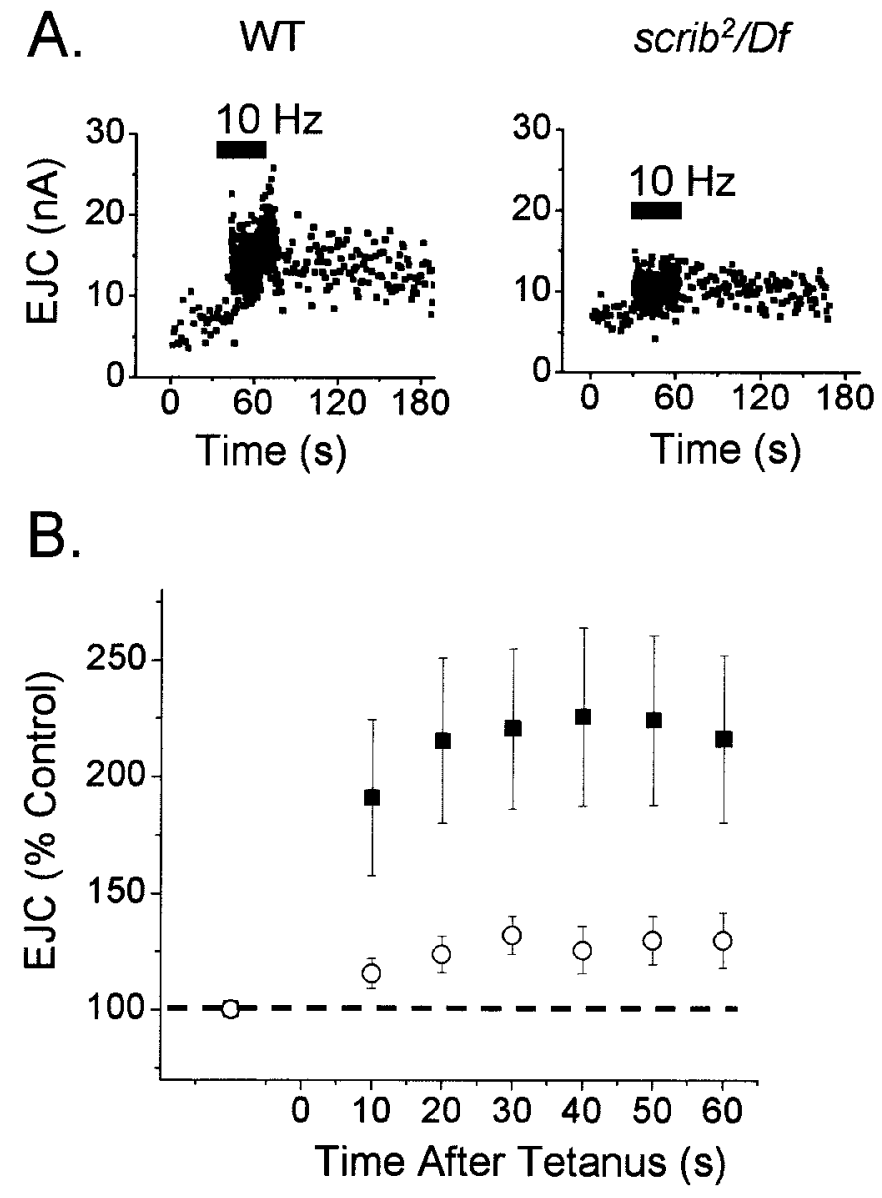

Figure 8. scrib mutants do not exhibit PTP. A, Representative recordings of wild type (left) and $s c r i b^{2 / D f}$ (right) using the PTP protocol. This protocol consisted of eliciting EJCs in a saline solution containing $0.4 \mathrm{~mm}$ $\mathrm{Ca}^{2+}$ and stimulating the segmental nerve at a frequency of $1 \mathrm{~Hz}$ for 30 sec, $10 \mathrm{~Hz}$ for $30 \mathrm{sec}$ (tetanus), and $1 \mathrm{~Hz}$ for the remainder of the experiment. Each symbol represents the amplitude of the EJC at that time point. The black bar represents the period of time in which the nerve was stimulated at $10 \mathrm{~Hz}$. B, The amplitude of the EJCs for the $30 \mathrm{sec}$ before tetanus was averaged and used as a control level. The average amplitude of the EJCs in $10 \mathrm{sec}$ intervals for $1 \mathrm{~min}$ after cessation of tetanus is represented for wild type ( filled squares; $n=7$ ) and $s c r i b^{2} / D f$ (open circles; $n=7)$. Dashed line represents normalized level of control $(100 \%)$.

recruitment of vesicles (Kuromi and Kidokoro, 2000). The vesicles are replenished to some degree by endocytosis of recently released vesicles. The increased density of vesicles in scrib mutants suggests that the defects in short-term plasticity are not likely a result of vesicle depletion, as is seen in the endocytotic mutant shibire, but more likely a result of the inability to rapidly recruit vesicles into the RRP. We tested this hypothesis by activity-dependent labeling of recycling vesicles using the styryl dye FM1-43 (Betz and Bewick, 1992).

For these studies, preparations were dissected in $0.1 \mathrm{mM} \mathrm{Ca}^{2+}$ HL-3 saline and subsequently depolarized for $2 \mathrm{~min}$ in HL-3 saline containing $90 \mathrm{~mm} \mathrm{~K}{ }^{+}$in the presence of $5 \mu \mathrm{M}$ FM1-43. After washing and fixing, samples were imaged by confocal microscopy. In wild type, strong FM1-43 fluorescence was observed at type I boutons (Fig. 9). This labeling paradigm has been shown to label what has been termed the exo/endo cycling pool (ECP) in Drosophila (Kuromi and Kidokoro, 1998), a pool that has been shown to contribute to both low- and high-frequency vesicle release (Kuromi and Kidokoro, 2000). 

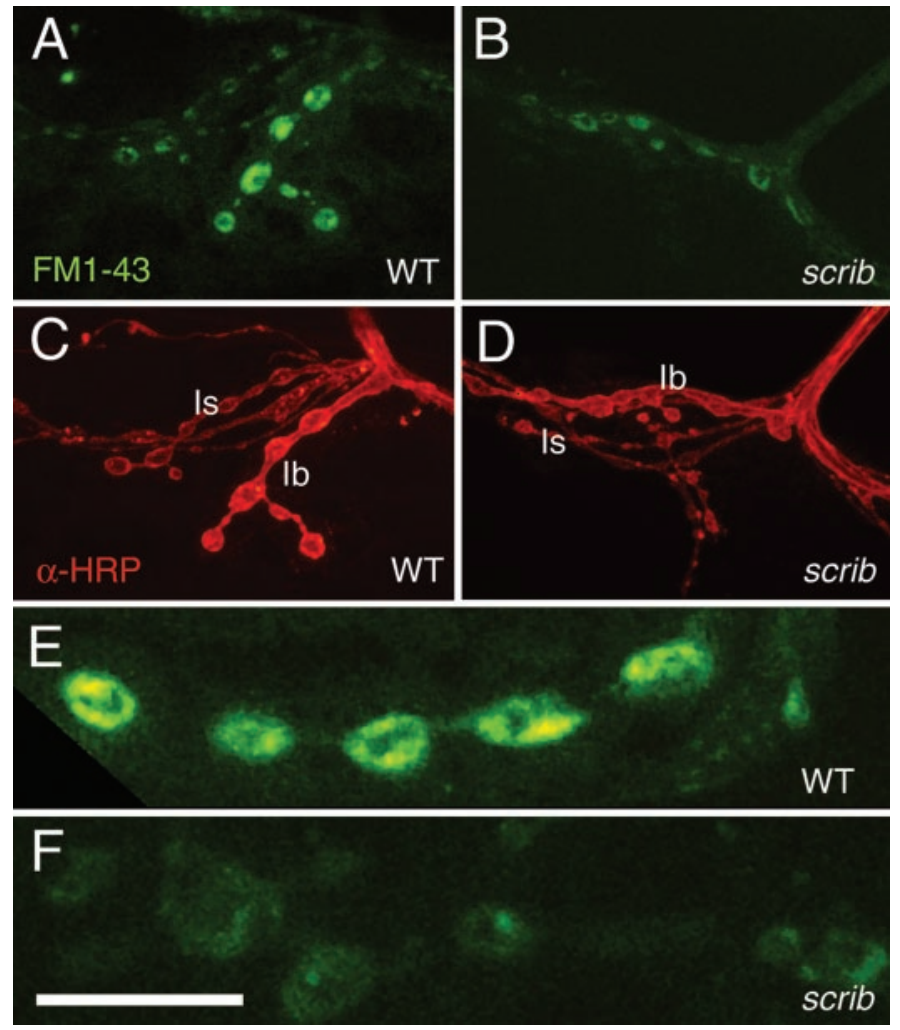

Figure 9. Vesicle recycling is impaired in scrib mutant larvae. Third instar larvae were incubated with FM1-43 for 2 min in the presence of 90 mM K ${ }^{+}$Stewart's HL-3 saline solution. The boutons were subsequently washed three times for $5 \mathrm{~min}$, fixed, and visualized using confocal microscopy. Representative micrographs show uptake of FM1-43 in wild-type $(A)$ and $s c r i b^{2} / D f(B)$ larvae. $C, D$, After FM1-43 labeling, preparations were stained with anti-HRP antibodies to show the location of the synaptic boutons displayed in $A$ and $B . E, F$, High-magnification views of FM1-43-labeled synaptic boutons in wild type $(E)$ and $s c r i b^{2 / D f}(F)$.

We found that this loading procedure resulted in fluorescence that was observed in a broad area at the periphery of synaptic boutons, whereas the central core of the boutons was devoid of fluorescence (Fig. 9). This is in agreement with previous reports showing a similar distribution of synaptic vesicles (Kuromi and Kidokoro, 2000). In $s c r i b^{2} / D f$ mutants we found a $54 \pm 5 \%$ reduction in FM1-43 fluorescence compared with wild-type levels ( $n=163$ boutons in $s c r i b^{2} / D f$ and 159 boutons in wild type). In addition, the distribution of the FM1-43 label was affected in the mutants, which showed a significantly larger percentage of boutons with diffuse FM1-43 throughout the bouton surface (62\% of boutons in $s c r i b^{2} / D f$ versus $10 \%$ of boutons in wild type). Thus, $s c r i b^{2} / D f$ mutants exhibit a decrease in activity-dependent endocytosis or altered distribution of endocytosed vesicles, or both. Because $s c r i b^{2} / D f$ larvae have very high synaptic vesicle densities in contrast to endocytotic mutants such as shibire and AP2, which show synaptic vesicle depletion (Chen et al., 1991; van der Bliek and Meyerowitz, 1991; Gonzalez-Gaitan and Jackle, 1997), we propose that the defect in scrib mutants arises from a defect in exocytosis that secondarily causes effects on vesicle distribution, rather than a specific defect in endocytosis or endocytotic sorting. Indeed, the number of vesicles contained in the ECP is known to affect further loading of synaptic vesicles (Kuromi and Kidokoro, 1998).

\section{DISCUSSION}

We have demonstrated here that the PDZ-containing protein Scrib substantially alters aspects of synapse ultrastructure. The effects include an increase in the number of synaptic vesicles, a decrease in the number of active zones, and a thickened basal lamina. Mutations in scrib also cause numerous functional changes, the most profound of which are the virtual elimination of short-term facilitation, an increase in synaptic depression, elimination of PTP, and altered vesicle recycling. These data indicate that Scrib has a prominent role in synapse function and, taking into account its multiple PDZ domains, is likely involved in the precise localization of proteins necessary for vesicle dynamics.

Facilitation is a $\mathrm{Ca}^{2+}$-dependent process that takes place in many synapses. This process is thought to result from residual $\mathrm{Ca}^{2+}$ accumulation, which acts on unidentified components of the exocytotic pathway (Zucker, 1999). The precise mechanism by which high-frequency stimulation may lead to an increase in quantal content is currently unknown; however, several possibilities exist, including an increase in the $p_{\mathrm{R}}$ of docked vesicles or an increase in the number of docked vesicles (Zucker, 1999). There are several mutations in Drosophila that have effects on facilitation and PTP, including dunce (cAMP-specific phosphodiesterase II) (Zhong and $\mathrm{Wu}, 1991)$, rutabaga $\left(\mathrm{Ca}^{2+} /\right.$ calmodulindependent adenylyl cyclase) (Zhong and $\mathrm{Wu}, 1991)$, volado ( $\alpha$ PS3-integrin) (Rohrbough et al., 2000), leonardo (a 14-3-3 protein family member) (Broadie et al., 1997), and latheo (an origin of replication complex protein) (Rohrbough et al., 1999). Of these mutants, scrib behaves most like rutabaga and latheo, in that both of these lack facilitation when baseline synaptic transmission is equivalent to wild type, and both have altered $\mathrm{Ca}^{2+}$ sensitivity of vesicle release, although the alterations in $\mathrm{Ca}^{2+}$ sensitivity are much more dramatic in both rutabaga and dunce mutants than in scrib mutants. It will be interesting to determine whether the localization of either of these proteins is altered in scrib mutants.

Three alternative models, or a combination of these, may contribute to the functional abnormalities observed in scrib mutants. One possibility is that the lack of facilitation observed at low $\mathrm{Ca}^{2+}$ in scrib mutants is caused by the inability of these synapses to recruit, transport, or convert vesicles into the RRP. This notion is supported by the FM1-43 studies, showing that endocytosis of vesicles in scrib mutants is greatly reduced, because endocytosis would be influenced by the amount of vesicles released as well as the number of vesicles already contained in the synapse (Kuromi and Kidokoro, 1998). In other words, the cellular machinery underlying facilitation may be intact in scrib mutants, but the inability to recruit additional vesicles may mask the expression of facilitation. Similarly, during high-frequency stimulation at higher $\mathrm{Ca}^{2+}$, a decreased capability to recruit vesicles to the RRP would result in increased magnitude of depression in scrib mutants.

A second model is based on evidence indicating the existence of a feedback mechanism at the Drosophila NMJ that operates to maintain the excitability of the muscle within a narrow range (Stewart et al., 1996; Marek et al., 2000; Paradis et al., 2001). Because of this compensatory mechanism, the decrease in number of active zones in scrib mutants may lead to a maximal increase in $p_{\mathrm{R}}$, thus maintaining EJC amplitude at low frequencies. Stimulation of the terminal at frequencies that in wild type lead to facilitation, however, would be unable to further increase $p_{\mathrm{R}}$ in scrib mutants, precluding further increases in the amplitude 
of the EJC. In other words, compensatory mechanisms in scrib mutants bring the mutant synapse to a facilitated state even at low-frequency stimulation, preventing a further increase in EJC amplitude at higher frequencies. The rapid depression observed in scrib mutants could be explained according to this model by the inability of the terminal to fulfill the increased demand for primed vesicles that is imposed by the increase in $p_{\mathrm{R}}$. In intact Drosophila larvae, motoneurons stimulate the muscles by firing trains of high-frequency action potentials (Budnik et al., 1990). In scrib mutants, loss of high-frequency responsiveness might lead to the buildup of synaptic vesicles, perhaps explaining the enhanced density of synaptic vesicle in scrib mutants. Thus this model is consistent with the electrophysiological and ultrastructural observations in the mutants.

A third possible mechanism involves altered buffering of neuronal $\mathrm{Ca}^{2+}$ levels. Cytoplasmic $\mathrm{Ca}^{2+}$ buffering is important in regulating synaptic vesicle release, especially during highfrequency stimulation (Dittman and Regehr, 1998; Stevens and Wesseling, 1998; Wang and Kaczmarek, 1998; Zucker and Regehr, 2002). A prevalent form of buffering $\mathrm{Ca}^{2+}$ at synapses is mitochondrial $\mathrm{Ca}^{2+}$ uptake, and this form of $\mathrm{Ca}^{2+}$ uptake has been shown to have effects on high-frequency synaptic firing patterns (Nguyen et al., 1997). The number of mitochondria in scrib boutons, however, was unchanged from wild type, making this possibility less likely. However, elimination or mislocalization of other critical $\mathrm{Ca}^{2+}$ buffering or sensing molecules may play a role in the defects seen in scrib synapses. Ultimately, the identification of Scrib binding partners may shed light on the precise mechanisms by which facilitation and sustained release are affected in scrib mutants.

Another defect in scrib mutant synapses was an abnormally thick basal lamina. The synaptic basal lamina has long been recognized as containing important elements for postsynaptic differentiation and for the clustering of neurotransmitter receptors (Sanes and Lichtman, 1999). The effects of Scrib on the basal lamina may reflect the inability of Scrib to selectively recruit synaptic components to their correct destination.

PDZ proteins are characterized by multiple modular sequences involved in protein-protein interactions and for this reason are frequently called scaffolding proteins (Sheng and Sala, 2001). These proteins bring together components necessary for certain cellular functions by binding to distinct partners through their multiple interaction domains. Scrib contains four PDZ domains and hence could form a multiprotein complex with at least four different proteins. Elucidation of the identity of these binding partners will be important in understanding the precise role that Scrib plays in synaptic vesicle dynamics. In epithelial cells it has been suggested that Scrib is involved in vesicle sorting (Bilder and Perrimon, 2000), an important mechanism involved in segregating transmembrane proteins in these cells. Another PDZ domaincontaining protein, EBP50, has also been shown to be involved in vesicular sorting, in particular, sorting of endocytotic vesicles involved in recycling $\beta_{2}$ adrenergic receptors (Cao et al., 1999). A similar mechanism, perhaps operating through a conserved cassette of proteins, may be altering vesicle dynamics at the synapse.

One might hypothesize, on the basis of the mislocalization of Scrib in $d l g$ mutants (Mathew et al., 2002), that by mutating $d l g$ one would see, in addition to a dlg-specific phenotype, the scrib phenotype as well. Indeed this seems to be the case in other cell types in which mutation of either $d l g$ or scrib causes similar phenotypes: formation of tumors and loss of cell polarity (Bilder and Perrimon, 2000). However, we have found that at the Dro- sophila NMJ the effects of scrib mutation are quite different from that of several $d l g$ mutants (Budnik et al., 1996). These differences may stem from the fact that residual synaptic Scrib is still present in $d g^{X I-2}$ mutants, although at a lower level, and hence the remaining Scrib may be sufficient to override the synaptic scrib phenotype. Interestingly, levels of synaptic Scrib have an opposite influence on the regulation of the number of active zones than do levels of synaptic Dlg. Although a decrease in Dlg levels in severe $d l g$ mutants causes an increase in active zone number, the same phenotype is observed by increasing Scrib levels. This observation is consistent with the notion that at synapses Scrib may negatively regulate Dlg function. This is in contrast to the observation in epithelial cells, where Dlg and Scrib appear to function in a similar manner during the determination of cell polarity and tumor suppression (Bilder and Perrimon, 2000). This may reflect the ability of Dlg and Scrib to bind different protein partners with different functions in the two cell types. Indeed, partners such as Fasciclin II bind to Dlg at synapses but are absent in epithelial cells (Thomas et al., 1997). Thus, the specific influence of scaffolding proteins in different cell types may be highly dependent on the availability of specific binding partners.

In conclusion, we have characterized a novel player in synapse formation and function. We also have introduced a relatively new function for a member of the PDZ protein family, regulation of synaptic vesicles. It will be important to understand the specific subset of proteins that interacts with Scrib, because this is likely the key to understanding how this protein is involved in synaptic vesicle regulation.

\section{REFERENCES}

Betz WJ, Bewick GS (1992) Optical analysis of synaptic vesicle recycling at the frog neuromuscular junction. Science 255:200-203.

Bilder D, Perrimon N (2000) Localization of apical epithelial determinants by the basolateral PDZ protein Scribble. Nature 403:676-680.

Bilder D, Li M, Perrimon N (2000) Cooperative regulation of cell polarity and growth by Drosophila tumor suppressors. Science 289:113-116.

Broadie K, Rushton E, Skoulakis EM, Davis RL (1997) Leonardo, a Drosophila 14-3-3 protein involved in learning, regulates presynaptic function. Neuron 19:391-402.

Budnik V (1996) Synapse maturation and structural plasticity at Drosophila neuromuscular junctions. Curr Opin Neurobiol 6:858-867.

Budnik V, Zhong Y, Wu CF (1990) Morphological plasticity of motor axons in Drosophila mutants with altered excitability. J Neurosci 10:3754-3768.

Budnik V, Koh YH, Guan B, Hartmann B, Hough C, Woods D, Gorczyca M (1996) Regulation of synapse structure and function by the Drosophila tumor suppressor gene dlg. Neuron 17:627-640.

Cao TT, Deacon HW, Reczek D, Bretscher A, von Zastrow M (1999) A kinase-regulated PDZ-domain interaction controls endocytic sorting of the beta2-adrenergic receptor. Nature 401:286-290.

Chen HJ, Rojas-Soto M, Oguni A, Kennedy MB (1998) A synaptic Ras-GTPase activating protein (p135 SynGAP) inhibited by CaM kinase II. Neuron 20:895-904.

Chen MS, Obar RA, Schroeder CC, Austin TW, Poodry CA, Wadsworth SC, Vallee RB (1991) Multiple forms of dynamin are encoded by shibire, a Drosophila gene involved in endocytosis. Nature 351:583-586.

Dittman JS, Regehr WG (1998) Calcium dependence and recovery kinetics of presynaptic depression at the climbing fiber to Purkinje cell synapse. J Neurosci 18:6147-6162.

Dodge Jr FA, Rahamimoff R (1967) Cooperative action of calcium ions in transmitter release at the neuromuscular junction. J Physiol (Lond) 193:419-432.

Garcia EP, Mehta S, Blair LA, Wells DG, Shang J, Fukushima T, Fallon JR, Garner CC, Marshall J (1998) SAP90 binds and clusters kainate receptors causing incomplete desensitization. Neuron 21:727-739.

Gonzalez-Gaitan M, Jackle H (1997) Role of Drosophila alpha-adaptin in presynaptic vesicle recycling. Cell 88:767-776.

Jia XX, Gorczyca M, Budnik V (1993) Ultrastructure of neuromuscular junctions in Drosophila: comparison of wild type and mutants with increased excitability. J Neurobiol 24:1025-1044.

Kim E, Sheng M (1996) Differential K+ channel clustering activity of PSD-95 and SAP97, two related membrane-associated putative guanylate kinases. Neuropharmacology 35:993-1000. 
Kim JH, Liao D, Lau LF, Huganir RL (1998) SynGAP: a synaptic RasGAP that associates with the PSD-95/SAP90 protein family. Neuron 20:683-691.

Koh YH, Popova E, Thomas U, Griffith LC, Budnik V (1999) Regulation of DLG localization at synapses by CaMKII-dependent phosphorylation. Cell 98:353-363.

Koh YH, Gramates LS, Budnik V (2000) Drosophila larval neuromuscular junction: molecular components and mechanisms underlying synaptic plasticity. Microsc Res Tech 49:14-25.

Kornau HC, Schenker LT, Kennedy MB, Seeburg PH (1995) Domain interaction between NMDA receptor subunits and the postsynaptic density protein PSD-95. Science 269:1737-1740.

Kuromi H, Kidokoro Y (1998) Two distinct pools of synaptic vesicles in single presynaptic boutons in a temperature-sensitive Drosophila mutant, shibire. Neuron 20:917-925.

Kuromi H, Kidokoro Y (2000) Tetanic stimulation recruits vesicles from reserve pool via a cAMP-mediated process in Drosophila synapses. Neuron 27:133-143.

Lahey T, Gorczyca M, Jia XX, Budnik V (1994) The Drosophila tumor suppressor gene dlg is required for normal synaptic bouton structure. Neuron 13:823-835.

Marek KW, Ng N, Fetter R, Smolik S, Goodman CS, Davis GW (2000) A genetic analysis of synaptic development: pre- and postsynaptic dCBP control transmitter release at the Drosophila NMJ. Neuron 25:537-547.

Mathew D, Gramates LS, Packard M, Thomas U, Bilder D, Perrimon N, Gorczyca M, Budnik V (2002) Recruitment of scribble to the synaptic scaffolding complex requires GUK-holder, a Novel DLG binding protein. Curr Biol 12:531-539.

Nguyen PV, Marin L, Atwood HL (1997) Synaptic physiology and mitochondrial function in crayfish tonic and phasic motor neurons. J Neurophysiol 78:281-294.

Niethammer M, Kim E, Sheng M (1996) Interaction between the C terminus of NMDA receptor subunits and multiple members of the PSD-95 family of membrane-associated guanylate kinases. J Neurosci 16:2157-2163.

Paradis S, Sweeney ST, Davis GW (2001) Homeostatic control of presynaptic release is triggered by postsynaptic membrane depolarization. Neuron 30:737-749.

Rohrbough J, Pinto S, Mihalek RM, Tully T, Broadie K (1999) latheo, a Drosophila gene involved in learning, regulates functional synaptic plasticity. Neuron 23:55-70.
Rohrbough J, Grotewiel MS, Davis RL, Broadie K (2000) Integrinmediated regulation of synaptic morphology, transmission, and plasticity. J Neurosci 20:6868-6878.

Sanes JR, Lichtman JW (1999) Development of the vertebrate neuromuscular junction. Annu Rev Neurosci 22:389-442.

Sheng M (2001) Molecular organization of the postsynaptic specialization. Proc Natl Acad Sci USA 98:7058-7061.

Sheng M, Sala C (2001) PDZ domains and the organization of supramolecular complexes. Annu Rev Neurosci 24:1-29.

Stevens CF, Wesseling JF (1998) Activity-dependent modulation of the rate at which synaptic vesicles become available to undergo exocytosis. Neuron 21:415-424.

Stewart BA, Atwood HL, Renger JJ, Wang J, Wu CF (1994) Improved stability of Drosophila larval neuromuscular preparations in haemolymph-like physiological solutions. J Comp Physiol [A] 175:179-191.

Stewart BA, Schuster CM, Goodman CS, Atwood HL (1996) Homeostasis of synaptic transmission in Drosophila with genetically altered nerve terminal morphology. J Neurosci 16:3877-3886.

Thomas U, Kim E, Kuhlendahl S, Koh YH, Gundelfinger ED, Sheng M, Garner CC, Budnik V (1997) Synaptic clustering of the cell adhesion molecule fasciclin II by discs-large and its role in the regulation of presynaptic structure. Neuron 19:787-799.

Thomas U, Ebitsch S, Gorczyca M, Koh YH, Hough CD, Woods D, Gundelfinger ED, Budnik V (2000) Synaptic targeting and localization of discs-large is a stepwise process controlled by different domains of the protein. Curr Biol 10:1108-1117.

Torroja L, Packard M, Gorczyca M, White K, Budnik V (1999) The Drosophila $\beta$-amyloid precursor protein homolog promotes synapse differentiation at the neuromuscular junction. J Neurosci 19:7793-7803.

van der Bliek AM, Meyerowitz EM (1991) Dynamin-like protein encoded by the Drosophila shibire gene associated with vesicular traffic. Nature 351:411-414.

Wang LY, Kaczmarek LK (1998) High-frequency firing helps replenish the readily releasable pool of synaptic vesicles. Nature 394:384-388.

Zhong Y, Wu CF (1991) Altered synaptic plasticity in Drosophila memory mutants with a defective cyclic AMP cascade. Science 251:198-201.

Zucker RS (1999) Calcium- and activity-dependent synaptic plasticity. Curr Opin Neurobiol 9:305-313.

Zucker RS, Regehr WG (2002) Short-term synaptic plasticity. Annu Rev Physiol 64:355-405. 